\title{
Millihertz quasi-periodic oscillations in 4U 1636-53 associated with bursts with positive convexity only
}

\author{
Ming Lyu ${ }^{1 \star}$, Mariano Méndez ${ }^{1}$, Diego Altamirano ${ }^{2}$ and Guobao Zhang ${ }^{3}$ \\ ${ }^{1}$ Kapteyn Astronomical Institute, University of Groningen, PO BOX 800, NL-9700 AV Groningen, the Netherlands \\ ${ }^{2}$ School of Physics and Astronomy, University of Southampton, Southampton, SO17 1BJ, UK \\ ${ }^{3}$ New York University Abu Dhabi, P.O. Box 129188, Abu Dhabi, United Arab Emirates
}

Accepted XXXX. Received XXXX; in original form XXXX

\begin{abstract}
We investigated the convexity of all type I X-ray bursts with millihertz quasi-periodic oscillations ( $\mathrm{mHz}$ QPOs) in 4U 1636-53 using archival observations with the Rossi X-ray Timing Explorer. We found that, at a $3.5 \sigma$ confidence level, in all 39 cases in which the $\mathrm{mHz}$ QPOs disappeared at the time of an X-ray burst, the convexity of the burst is positive. The convexity measures the shape of the rising part of the burst light curve and, according to recent models, it is related to the ignition site of bursts on the neutronstar surface. This finding suggests that in $4 \mathrm{U} 1636-53$ these 39 bursts and the marginally-stable nuclear burning process responsible for the $\mathrm{mHz}$ QPOs take place at the neutron-star equator. This scenario could explain the inconsistency between the high accretion rate required for triggering $\mathrm{mHz}$ QPOs in theoretical models and the relatively low accretion rate derived from observations.
\end{abstract}

Key words: X-rays: binaries; stars: neutron; accretion, accretion discs; Xrays: bursts; X-rays: individual: $4 \mathrm{U} 1636-53$

\section{INTRODUCTION}

Nearly half of the accreting neutron stars in low-mass X-ray binaries show Type I X-ray bursts (e.g., in’t Zand et al. 2004; Liu et al. 2007; Galloway et al. 2008). These bursts are 
due to unstable thermonuclear burning of accumulated hydrogen and helium on the surface of the neutron star (e.g., Fujimoto et al. 1981). In the last decade another observational phenomenon connected to nuclear burning on the neutron-star surface has been discovered. Revnivtsev et al. (2001) reported the first detection of quasi-periodic oscillations (QPOs) in the millihertz $(\mathrm{mHz})$ range in three neutron-star low-mass X-ray binaries (NS LMXBs): 4U 1608-52, 4U 1636-53, and Aql X-1. Besides the low frequency range between 7 and 9 $\mathrm{mHz}$, the $\mathrm{mHz}$ QPOs show some unique properties compared to other types of QPOs in NS LMXBs: The $\mathrm{mHz}$ QPOs happen only within a particular luminosity range, $L_{2-20 \mathrm{keV}} \simeq$ $(5-11) \times 10^{36} \operatorname{ergs~s}^{-1}$, and are stronger at low photon energies $(E<5 \mathrm{keV})$ (Revnivtsev et al. 2001; Altamirano et al. 2008).

Altamirano et al. (2008) found that the frequency of the $\mathrm{mHz}$ QPO in $4 \mathrm{U} 1636-53$ decreased systematically with time until the QPO became undetectable at the time of a type I X-ray burst when the source was in the transition between hard and soft state usually seen in these systems. Linares et al. (2010) found $\mathrm{mHz}$ QPOs in the neutron-star transient IGR J17480-2446 in the globular cluster Terzan 5. These mHz QPOs showed some different properties with respect to the ones in other sources: The QPO frequency was relatively low, always below $4.5 \mathrm{mHz}$, and the persistent source luminosity at the time the QPOs appeared was high, $L_{2-50 \mathrm{keV}} \sim 10^{38} \mathrm{erg} \mathrm{s}^{-1}$. Furthermore, Linares et al. (2012) found a smooth evolution between X-ray bursts and $\mathrm{mHz}$ QPOs in IGR J17480-2446 as the luminosity of the source changed during the outburst, which has never been observed in other $\mathrm{mHz} \mathrm{QPO}$ sources.

The above observational findings suggest a different origin of the $\mathrm{mHz}$ QPOs from other kinds of QPOs (e.g., van Straaten et al. 2002, 2005; van der Klis 2006; Altamirano et al. 2008) in NS LMXBs. Revnivtsev et al. (2001) speculated that a special mode of nuclear burning on the neutron-star surface may be responsible for the $\mathrm{mHz}$ QPOs. Heger et al. (2007) proposed that the $\mathrm{mHz}$ QPOs could be a consequence of marginally stable nuclear burning of Helium on the neutron-star surface. The model of Heger et al. (2007) is able to explain the characteristic time scale of $\sim 2$ minutes of the $\mathrm{mHz}$ QPOs, and predicts that the QPOs should occur only in a very narrow range of X-ray luminosity. However, the accretion rate at which the $\mathrm{mHz}$ QPOs are predicted in the model is close to the Eddington rate, up to one order of magnitude higher than the one implied by the X-ray luminosity at which $\mathrm{mHz}$ QPOs were observed. To bring the models and observations into agreement, Heger et al. (2007) proposed that the local accretion rate in the burning layer where the QPOs 
happen can be higher than the global accretion rate. Keek et al. (2009) found that turbulent chemical mixing of the fuel, together with a higher heat flux from the crust, can explain the observed accretion rate at which $\mathrm{mHz}$ QPOs are seen. Furthermore, Altamirano et al. (2008) and Keek et al. (2009) suggested that the cooling process of the layer where the $\mathrm{mHz}$ QPOs happen may be responsible for the frequency drift of the QPOs before X-ray bursts. Keek et al. (2014) explored the influence of the fuel composition and nuclear reaction rates on the $\mathrm{mHz}$ QPOs, and concluded that no allowed variation in the composition and the reaction rate is able to trigger the $\mathrm{mHz}$ QPOs at the observed accretion rates.

Lyu et al. (2015) investigated the relation between the frequency of the $\mathrm{mHz}$ QPOs and the temperature of the neutron-star surface in 4U 1636-53 using XMM-Newton and simultaneous RXTE observations, and they found that there was no significant correlation, which is different from theoretical predictions. Besides, Lyu et al. (2015) found that all seven X-ray bursts associated with $\mathrm{mHz}$ QPOs in this source were bright, energetic and short, indicating a potential connection between the $\mathrm{mHz}$ QPOs and He-rich X-ray bursts.

Cooper \& Narayan (2007) found that the latitude at which type I X-ray bursts ignite on the neutron star surface depends on the accretion rate. Later, Maurer \& Watts (2008) simulated the influence of ignition latitude, accretion rate and neutron-star rotation on the shape of the rising phase of type I X-ray bursts. They found that bursts that ignite at the equator always have positive convexity, whereas bursts that ignite at high latitude have both positive and negative convexity. The convexity measures the shape of the rising part of the burst light curve, and it is defined as the integrated area of the burst light curve above (positive convexity) or below (negative convexity) a straight line drawn from the start to the peak of the burst. Recently, Mahmoodifar \& Strohmayer (2015) further confirmed that the rising part of the light curve of bursts is more concave when ignition starts near the pole compared to when it starts near the equator. Thus, the convexity of an X-ray burst provides information about the ignition site of unstable nuclear burning on the neutron-star surface. The fact that $\mathrm{mHz}$ QPOs are closely related to type I X-ray bursts opens up the possibility to study the origin and physics of marginally stable nuclear burning on the neutron-star surface, by investigating $\mathrm{mHz}$ QPOs and type I X-ray bursts together. In this paper we focus on the possible connection between $\mathrm{mHz}$ QPOs and the convexity of type I X-ray bursts to explore the site on the neutron-star surface at which the marginally stable nuclear burning ignites. 


\section{OBSERVATIONS AND DATA REDUCTION}

We analysed all available data of 4U 1636-53 from the Proportional Counter Array (PCA; Jahoda et al. 2006) on board of the Rossi X-ray Timing Explorer (RXTE) using the Heasoft 6.16. An RXTE observation typically covers 1 to 5 consecutive 90-minute satellite orbits. Usually, an orbit contains between 1 and $5 \mathrm{ks}$ of useful data separated by 1-4 ks data gaps; on rare occasions the visibility windows were such that RXTE continuously observed the source for up to $\sim 27 \mathrm{ks}$. This means that our datasets consist of continuous data segments of lengths between 0.3 and $27 \mathrm{ks}$.

We used 1-s resolution event mode PCA light curves in the $\sim 2-5 \mathrm{keV}$ range (where the $\mathrm{mHz}$ QPOs are the strongest, see Altamirano et al. 2008) and searched for periodicities in each of the gap-free segments separately using Lomb-Scargle periodograms (Lomb 1976; Scargle 1982; Press et al. 2002). In those cases where more than one Type I X-ray burst was detected, we searched for $\mathrm{mHz}$ QPOs before, after and in-between bursts. We only report those detections that are at least $3 \sigma$ significant as estimated using the method outlined in Press et al. (2002). When undetected, It is difficult to estimate a general and/or meaningful upper limit on the fractional rms amplitude of the $\mathrm{mHz}$ QPOs before an X-ray burst. The reasons could be many: data-gaps just before the burst, or the segment before the burst is too short to detect the QPO significantly, or there is a reduced number of PCUs during that observation. In the few cases without the above problems, we estimated $3 \sigma$ upper limits as low as $0.4 \%$ rms in the $2-5 \mathrm{keV}$ range.

We investigated all X-ray bursts of $4 \mathrm{U} 1636-53$ detected by the PCA/RXTE. For this we produced 0.25-s light curve from the Standard-1/Event data and searched for X-ray bursts

in these light curves following the procedure described in Zhang et al. (2011). In order to study the shape and time-scale of the bursts rise, we extracted the bursts light curves from the PCA data with 0.125-s time resolution. To describe the shape of the burst rising phase quantitatively, we used the convexity, $\mathcal{C}$, parameter in our analysis (Maurer \& Watts 2008). The convexity describes the curvature of the light-curve rise, and it quantifies whether the curve is convex $(\mathcal{C}>0)$ or concave $(\mathcal{C}<0)$. We used the same method as in Maurer \& Watts (2008) to calculate the convexity in the burst light curve of the full PCA energy band; Since different bursts have different durations and peak intensities, we normalised the light curves and time axes so that, from the start to the peak, each burst rises from 0 to 10 normalised intensity units within 0 to 10 normalised time units, and we calculated the convexity in the 
time interval in which the light curve of the burst rises from 1 to 9 normalised intensity units, for more details of the calculation please refer to Zhang et al. (2016). We also calculated the rising time of each burst, defined as the time interval at the beginning of a burst during which the flux in the light curve is between $10 \%$ and $90 \%$ of the flux at the peak of the burst.

\section{RESULTS}

We detected 207 cases of $\mathrm{mHz}$ QPOs and 371 X-ray bursts in the whole RXTE archive. We excluded, and did not analyse further, those bursts that showed at least one of the following characteristics: (i) The burst light curve was incomplete, (ii) the burst light curve had multiple peaks, or (iii) the burst was very weak and hence the light curve was very noisy. We further excluded the superburst in this source (Wijnands 2001; Strohmayer \& Markwardt 2002). We were then left with 305 burst with a complete and smooth profile. We considered that a $\mathrm{mHz} \mathrm{QPO}$ and an X-ray burst are associated if, in an observation, there is a $\mathrm{mHz}$ QPO that ends at the same time that an X-ray burst happens. In the rest of the paper we only considered those cases in which the $\mathrm{mHz}$ QPOs are associated to an X-ray burst. We detected both $\mathrm{mHz}$ QPOs and an associated type I X-ray bursts in 39 observations; the QPOs in these observations always disappeared at the time when the associated X-ray burst appeared. In Figure 1 we show the distribution of the convexity of all type I X-ray bursts and the distribution of the convexity of those bursts that are associated with $\mathrm{mHz}$ QPOs in $4 \mathrm{U}$ 1636-53. The distribution of the convexity of all bursts is symmetric, with 252 and 53 of them having, respectively, positive and negative convexity. The distribution can be well fitted with a Gaussian function $(\mathrm{R}$-square=0.976) with a mean convexity of $12.3 \pm 1.2$ (95\% confidence level) and a standard deviation of $12.6 \pm 1.2$. For the 39 bursts associated with $\mathrm{mHz}$ QPOs, the convexities are always positive. We list the convexities of these 39 bursts in the Table 1. We found no case in our sample of a $\mathrm{mHz}$ QPO that is associated with a burst with negative convexity. In a few observations there is a second burst a few thousand seconds after the burst that is directly associated with the $\mathrm{mHz} \mathrm{QPO}$; in these cases we found that the convexity of the second burst can be either positive or negative. Furthermore, we found that the observed continuum flux ranges from $1.9 \times 10^{-9} \mathrm{ergs} \mathrm{cm}^{-2}$ $\mathrm{s}^{-1}$ to $5 \times 10^{-9} \mathrm{ergs} \mathrm{cm}^{-2} \mathrm{~s}^{-1}$ for bursts with $\mathrm{mHz}$ QPOs, and from $1.2 \times 10^{-9} \mathrm{ergs} \mathrm{cm}^{-2} \mathrm{~s}^{-1}$ to $8.2 \times 10^{-9}$ ergs $\mathrm{cm}^{-2} \mathrm{~s}^{-1}$ for bursts without $\mathrm{mHz}$ QPOs. The K-S test probability that the 


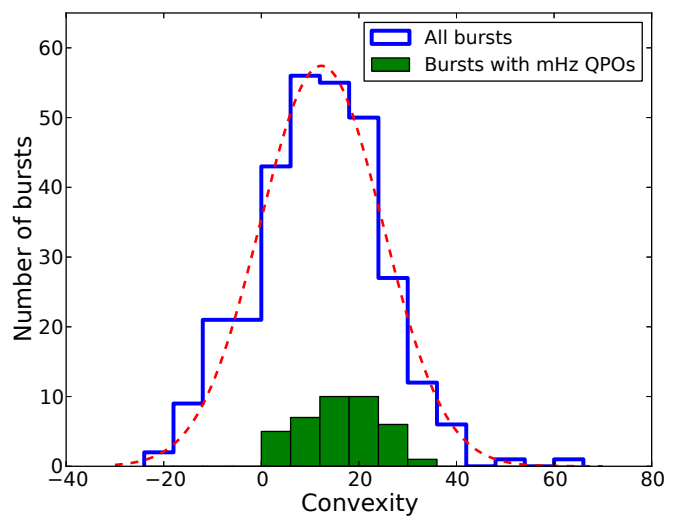

Figure 1. Distribution of the convexities of all X-ray bursts (blue line) and the bursts with $\mathrm{mHz}$ QPOs (filled green bars) in $4 \mathrm{U}$ 1636-53. The red dashed line in the plot corresponds to the best-fitting Gaussian curve to the the convexity distribution of all bursts.

above two samples come from the same parent population is $\mathrm{P}_{K-S}=0.0083$, which indicates that the distribution of the persistent flux of the two samples is marginally different.

The results shown in Figure 1 suggest that there is a relation between the presence of the $\mathrm{mHz}$ QPOs and the convexity of the associated burst. In order to quantify this, we calculated the probability, $\mathrm{P}_{39}$, of selecting 39 random bursts from the distribution of the convexity of all bursts in 4U 1636-53 (see Figure 1), and getting only bursts with positive convexity. Since the convexity can either be positive or negative, we can estimate this probability from the binomial distribution, where the probability of success (where success means $\mathcal{C}>0$ ) is $P=252 / 305=0.826$. The probability is then $\mathrm{P}_{39}=0.826^{39}=5.8 \times 10^{-4}$.

We also used the distribution in Figure 1 to simulate $10^{6}$ sets of 39 convexities, and counted the number of trials, $\mathrm{N}_{+}$, in which all 39 convexities were positive. We found that $\mathrm{N}_{+}=594$, corresponding to a probability of $5.9 \times 10^{-4}$, consistent with the calculation above.

In Figure 2 we show the distribution of the rising time of all X-ray bursts in 4U 1636-53. The rising time ranges from $0.4 \mathrm{~s}$ to $23 \mathrm{~s}$ and follows a bimodal distribution with peaks at $\sim 1 \mathrm{~s}$ and $\sim 3 \mathrm{~s}$, respectively. In Figure 3 we show the rising time vs. convexity of all bursts (blue snow symbols) and those bursts with $\mathrm{mHz}$ QPOs (red stars). The vertical line in this Figure is at a convexity of zero, while the horizontal line is at a rising time equal to $2 \mathrm{~s}$; the latter is approximately the value at which the distribution of rising times in Figure 2 shows a local minimum. It is apparent that all bursts with $\mathrm{mHz}$ QPOs are located on the lower right corner of this Figure: All bursts with $\mathrm{mHz}$ QPO have positive convexity and, except for one case, they all have rising times shorter than 2 s. From this Figure it is also apparent that not all bursts in that part of the diagram show $\mathrm{mHz}$ QPOs. 


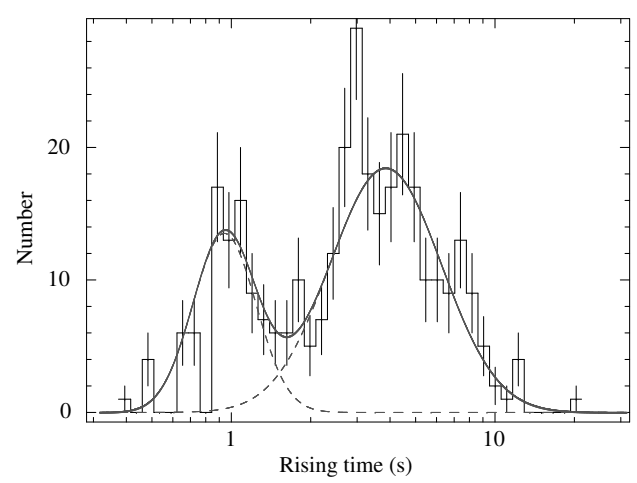

Figure 2. Distribution of the rising time of X-ray bursts in $4 \mathrm{U} 1636-53$. We used the dashed-lines to show the two best-fitted gaussians to the histogram, and the sum of the two Gauss components is shown as the black curve in the plot.

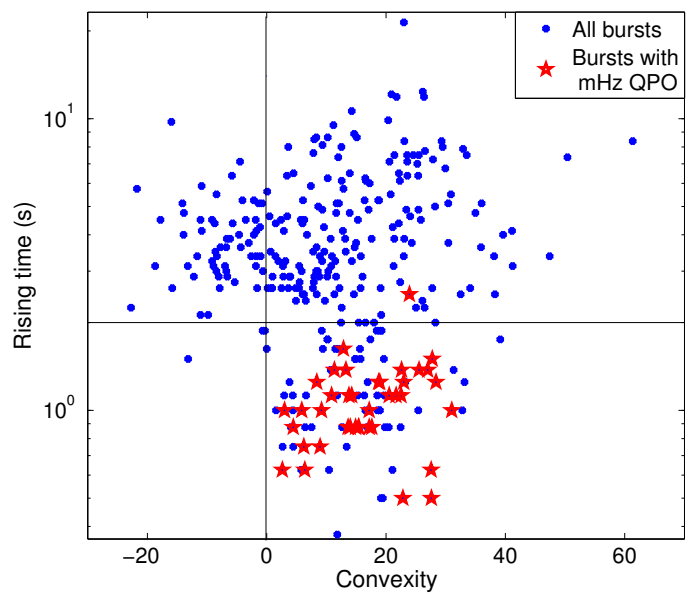

Figure 3. Rising time vs. convexity of X-ray bursts (blue snow symbols) and the bursts with mHz QPOs (red stars) in $4 \mathrm{U}$ 1636-53. The vertical and horizontal line in the plot corresponds to a convexity equal to 0 and a rising time equal to $2 \mathrm{~s}$.

\section{DISCUSSION}

Using data from the full RXTE archive we found that all type I X-ray bursts associated with the $\mathrm{mHz}$ QPOs (39 in total) in 4U 1636-53 have positive convexity. We did not find a single case in our sample of an X-ray burst with negative convexity associated to a $\mathrm{mHz}$ QPO. The probability that this happens only by chance is less than $6 \times 10^{-4}$, corresponding to a significance level of $\sim 3.5 \sigma$.

Using numerical simulations of the propagation of a burning front on the neutron-star surface, Maurer \& Watts (2008) found that bursts that ignite at the equator always have positive convexity, whereas bursts that ignite at high latitude have both positive and negative convexity. Mahmoodifar \& Strohmayer (2015) confirmed this result in their simulations, and also found that the rising time of bursts that ignite at the equator is short, whereas the rising time is both short or long for bursts that ignite at high latitudes (see also Maurer \& Watts 2008). In Table 2 we summarise the results of Maurer \& Watts (2008) and Mahmoodifar \& 
Table 1. List of the convexities of the 39 bursts associated with $\mathrm{mHz}$ QPOs in $4 \mathrm{U} 1636-53$. The continuum flux is from 2 to $50 \mathrm{keV}$ in unit of $10^{-9} \mathrm{ergs}^{-2} \mathrm{~s}^{-1}$. The convexity error here is at $1-\sigma$ significance level.

\begin{tabular}{|c|c|c|c|c|c|}
\hline ObsId & Star time of burst & End time of burst & Convexity & Rising time (s) & Absorbed continuum flux \\
\hline 10088-01-08-030 & 50448.73395 & 50448.73699 & $11.3 \pm 1.1$ & 1.4 & 4.8 \\
\hline 30053-02-02-02 & 51044.48934 & 51044.48976 & $13.3 \pm 1.2$ & 1.4 & 3.9 \\
\hline 40028-01-02-00 & 51236.36632 & 51236.36671 & $17.2 \pm 1.6$ & 1.0 & 4.1 \\
\hline 40028-01-04-00 & 51297.07198 & 51297.07243 & $4.5 \pm 1.5$ & 0.9 & 4.6 \\
\hline $40028-01-08-00$ & 51347.98825 & 51347.98866 & $25.5 \pm 1.5$ & 1.4 & 5.0 \\
\hline 40031-01-01-06 & 51350.79575 & 51350.79613 & $12.9 \pm 1.2$ & 1.6 & 3.8 \\
\hline $40028-01-15-00$ & 51710.21233 & 51710.21290 & $6.4 \pm 2.1$ & 0.6 & 4.3 \\
\hline $40028-01-19-00$ & 51768.98081 & 51768.98125 & $14.2 \pm 1.6$ & 1.1 & 3.8 \\
\hline $40028-01-20-00$ & 51820.98111 & 51820.98157 & $5.9 \pm 1.3$ & 1.0 & 3.7 \\
\hline 50030-02-05-00 & 51942.10024 & 51942.10065 & $3.0 \pm 1.4$ & 1.0 & 4.1 \\
\hline 50030-02-09-000 & 52004.71326 & 52004.71366 & $8.4 \pm 1.3$ & 1.3 & 4.1 \\
\hline $50030-02-10-00$ & 52029.22818 & 52029.22864 & $10.9 \pm 1.3$ & 1.1 & 3.1 \\
\hline 60032-01-02-00G & 52075.13477 & 52075.13512 & $27.6 \pm 2.8$ & 0.5 & 2.3 \\
\hline 60032-01-12-000 & 52182.61618 & 52182.61667 & $13.6 \pm 1.5$ & 0.9 & 2.7 \\
\hline 60032-01-14-01 & 52214.31827 & 52214.31882 & $18.9 \pm 1.4$ & 1.3 & 3.2 \\
\hline 60032-01-18-00G & 52273.69081 & 52273.69130 & $15.4 \pm 1.2$ & 0.9 & 2.0 \\
\hline $60032-01-20-000$ & 52283.01851 & 52283.01896 & $23.0 \pm 2.0$ & 1.3 & 2.3 \\
\hline $60032-01-20-01$ & 52283.53362 & 52283.53417 & $27.7 \pm 1.2$ & 1.5 & 2.4 \\
\hline 60032-05-01-00 & 52286.05404 & 52286.05451 & $2.6 \pm 2.4$ & 0.6 & 1.9 \\
\hline 60032-05-02-00 & 52286.55466 & 52286.55519 & $17.6 \pm 1.7$ & 0.9 & 2.0 \\
\hline 60032-05-04-00 & 52287.52190 & 52287.52233 & $9.2 \pm 1.7$ & 1.0 & 2.0 \\
\hline $60032-05-06-00$ & 52288.51431 & 52288.51476 & $27.0 \pm 1.5$ & 1.4 & 2.1 \\
\hline 60032-05-07-00 & 52288.97438 & 52288.97489 & $6.2 \pm 2.0$ & 0.8 & 1.9 \\
\hline 60032-05-07-01 & 52289.29282 & 52289.29320 & $15.4 \pm 2.6$ & 0.9 & 1.9 \\
\hline 60032-05-09-00 & 52289.97694 & 52289.97737 & $17.2 \pm 2.8$ & 0.9 & 2.1 \\
\hline $60032-05-18-00$ & 52390.21340 & 52390.21392 & $23.9 \pm 1.2$ & 2.5 & 3.0 \\
\hline $60032-05-23-000$ & 52646.77066 & 52646.77097 & $14.0 \pm 0.8$ & 0.9 & 2.4 \\
\hline $91024-01-30-10$ & 53688.95191 & 53688.95234 & $14.9 \pm 1.8$ & 0.9 & 4.3 \\
\hline $91152-05-02-00$ & 53919.07399 & 53919.07437 & $18.8 \pm 1.5$ & 1.3 & 4.0 \\
\hline $92023-01-29-10$ & 54050.90204 & 54050.90238 & $22.8 \pm 3.5$ & 0.5 & 2.9 \\
\hline $92023-01-31-10$ & 54054.24902 & 54054.24948 & $20.5 \pm 1.5$ & 1.1 & 2.9 \\
\hline 70036-01-02-010 & 54271.04381 & 54271.04432 & $13.8 \pm 0.8$ & 1.1 & 3.1 \\
\hline $70036-01-02-00$ & 54272.09180 & 54272.09229 & $27.6 \pm 2.9$ & 0.6 & 3.2 \\
\hline 93091-01-01-000 & 54371.71897 & 54371.71937 & $28.4 \pm 1.8$ & 1.3 & 2.0 \\
\hline $93087-01-24-10$ & 54522.68638 & 54522.68680 & $21.7 \pm 1.7$ & 1.1 & 2.5 \\
\hline 93091-01-02-00 & 54523.57841 & 54523.57893 & $8.9 \pm 2.2$ & 0.8 & 2.8 \\
\hline 93087-01-04-20 & 54678.26783 & 54678.26838 & $22.6 \pm 2.0$ & 1.4 & 2.5 \\
\hline 94310-01-01-00 & 54904.83290 & 54904.83362 & $22.5 \pm 1.6$ & 1.1 & 2.6 \\
\hline $94310-01-03-000$ & 55079.21966 & 55079.22008 & $31.0 \pm 1.7$ & 1.0 & 2.4 \\
\hline
\end{tabular}

Strohmayer (2015), statements $\mathbf{1 a}$ and $\mathbf{1 b}$, together with our own findings, statements $\mathbf{2 a}$ and $\mathbf{2 b}$. The last row in that Table shows the statements, 3, that follow logically from either the $a$ or the $b$ statements.

Bursts with short rising time are likely fuelled by Helium (Fujimoto et al. 1981). The apparent connection between $\mathrm{mHz}$ QPO and bursts with short rising time (Figure 3 ) suggests the possibility that $\mathrm{mHz}$ QPOs are due to marginally-stable nuclear burning of Helium on the neutron-star surface (Heger et al. 2007). However, there are as many bursts with a short rising time without $\mathrm{mHz} \mathrm{QPO}$ as with $\mathrm{mHz}$ QPO (lower right corner of Figure 3), which indicates that marginally-stable Helium burning can not be the only reason for the presence of $\mathrm{mHz}$ QPOs. 
Table 2. Properties of X-ray bursts.

\begin{tabular}{|c|c|c|c|}
\hline \multicolumn{4}{|c|}{ 1. Results from simulations: } \\
\hline a. Low-latitude ignition & $\Longrightarrow$ & $\mathcal{C}>0$ & $(1,2)$ \\
\hline High-latitude ignition & $\Longrightarrow$ & $\mathcal{C}>0$ or $\mathcal{C}<0$ & \\
\hline & $\cdots$ & & \\
\hline b. Low-latitude ignition & $\Longrightarrow$ & Short rising time & $(1,2)$ \\
\hline High-latitude ignition & $\Longrightarrow$ & Long/Short rising time & \\
\hline \multicolumn{4}{|c|}{ 2. Results from observations: } \\
\hline a. $\mathrm{mHz}$ QPOs & $\Longrightarrow$ & $\mathcal{C}>0$ & $(3)$ \\
\hline no $\mathrm{mHz}$ QPOs & $\Longrightarrow$ & $\mathcal{C}>0$ or $\mathcal{C}<0$ & \\
\hline b. $\mathrm{mHz}$ QPOs & $\stackrel{\cdots}{\Longrightarrow}$ & Short rising time & (3) \\
\hline no $\mathrm{mHz}$ QPOs & $\Longrightarrow$ & Long/Short rising time & \\
\hline \multicolumn{4}{|c|}{ 3. The statements $a$ or $b$ are logically equivalent to: } \\
\hline $\mathrm{mHz}$ QPOs & $\Longrightarrow$ & Low-latitude ignition & \\
\hline no $\mathrm{mHz}$ QPOs & $\Longrightarrow$ & Low-/High-latitude ignition & \\
\hline
\end{tabular}

From Table 2 we can conclude that all the 39 bursts with positive convexity and short rising time that are associated with $\mathrm{mHz}$ QPOs ignited at the neutron-star equator. For, if bursts associated with $\mathrm{mHz}$ QPOs ignited anywhere on the neutron-star surface (therefore these 39 bursts would correspond to cases of positive convexity and either low- or highlatitude ignition in the analysis of Maurer \& Watts (2008)), we would have expected to see also cases of $\mathrm{mHz}$ QPOs associated with bursts with negative convexity in our sample. While in this scenario bursts with positive convexity but no associated $\mathrm{mHz}$ QPO would have in principle ignited at high latitudes, some of them may also have ignited at the equator if, for instance, those bursts happened at an accretion rate in which marginally stable nuclear burning would not be at work (e.g., Heger et al. 2007). Also, in some cases a QPO might be present just before an X-ray burst, but we are unable to detect it either because we do not have enough data before a burst (e.g., if the data segment before the burst was too short), or because the data are not of sufficient quality to detect the QPO significantly (e.g., if some PCU detectors were not operating during that observation).

The simplest scenario that follows from this is that the marginally-stable burning (that produces the QPO) and the unstable burning (that produces the burst) take place at the same physical location. There should still be enough fuel at the equator to trigger a burst after the $\mathrm{mHz}$ QPOs if, similar to the case of unstable burning at high luminosity (e.g., van Paradijs et al. 1988; Muno et al. 2000; Cornelisse et al. 2003; Heger et al. 2007), marginallystable burning consumes only a fraction of the fuel on the surface of the neutron star. We cannot discard, however, more complex scenarios in which the sites of marginally-stable and 
unstable burning are physically disconnected, $\mathrm{mHz}$ QPOs and bursts with positive convexity happen at any latitude, but some other mechanism ensure that mHz QPOs and bursts with positive convexity are causally connected.

Fujimoto et al. (1981) proposed that the thermal stability and burst ignition of a neutron star actually depends on the accretion rate per unit area, $\dot{m}$, instead of the global accretion rate. The quantity $\dot{m}$ needs not to be the same everywhere on the neutron-star surface (e.g., Fujimoto et al. 1981; Bildsten 1998). During accretion, the infalling matter first reaches the equator and then spreads over the whole surface of the neutron star, therefore $\dot{m}$ will be higher at the equator than at high latitudes. If the $\mathrm{mHz}$ QPOs happen at the equator, the local accretion rate per unit area, $\dot{m}$, would also be the key parameter that determines whether marginally stable nuclear burning on the neutron-star surface takes place: when nuclear burning occurs around the equator, $\dot{m}$ is high enough to trigger the $\mathrm{mHz}$ QPOs, while there are no $\mathrm{mHz}$ QPOs when the nuclear burning happens at high latitudes where $\dot{m}$ is below the threshold value to trigger the marginally-stable nuclear burning process. The fact that the distribution of the persistent flux of observations with and without $\mathrm{mHz} \mathrm{QPOs}$ is consistent with being the same further enhances the argument that it is the local accretion rate $\dot{m}$ that triggers $\mathrm{mHz}$ QPOs. This picture is similar to the one proposed in Heger et al. (2007) in which the accreted fuel that is responsible for the marginally-stable nuclear burning is confined at a certain burning depth, where the local accretion rate could be much higher than the global accretion rate. This scenario is able to bridge the gap between the high accretion rate required for triggering the $\mathrm{mHz}$ QPOs in the models and the relatively low accretion rate implied from observations.

\section{ACKNOWLEDGMENTS}

This research has made use of data obtained from the High Energy Astrophysics Science Archive Research Center (HEASARC), provided by NASA's Goddard Space Flight Center. This research made use of NASA's Astrophysics Data System. LM is supported by China Scholarship Council (CSC), grant number 201208440011. DA acknowledges support from the Royal Society. 


\section{REFERENCES}

Altamirano D., van der Klis M., Méndez M., Jonker P. G., Klein-Wolt M., Lewin W. H. G., 2008, ApJ, 685, 436

Altamirano D., van der Klis M., Wijnands R., Cumming A., 2008, ApJ, 673, L35

Bildsten L., 1998, in Buccheri R., van Paradijs J., Alpar A., eds, NATO Advanced Science Institutes (ASI) Series C Vol. 515 of NATO Advanced Science Institutes (ASI) Series C, Thermonuclear Burning on Rapidly Accreting Neutron Stars. p. 419

Cooper R. L., Narayan R., 2007, ApJ, 657, L29

Cornelisse R., in't Zand J. J. M., Verbunt F., Kuulkers E., Heise J., den Hartog P. R., Cocchi M., Natalucci L., Bazzano A., Ubertini P., 2003, A\&A, 405, 1033

Fujimoto M. Y., Hanawa T., Miyaji S., 1981, ApJ, 247, 267

Galloway D. K., Muno M. P., Hartman J. M., Psaltis D., Chakrabarty D., 2008, ApJS, 179,360

Heger A., Cumming A., Woosley S. E., 2007, ApJ, 665, 1311

in’t Zand J., Verbunt F., Heise J., Bazzano A., Cocchi M., Cornelisse R., Kuulkers E., Natalucci L., Ubertini P., 2004, Nuclear Physics B Proceedings Supplements, 132, 486

Jahoda K., Markwardt C. B., Radeva Y., Rots A. H., Stark M. J., Swank J. H., Strohmayer T. E., Zhang W., 2006, ApJS, 163, 401

Keek L., Cyburt R. H., Heger A., 2014, ApJ, 787, 101

Keek L., Langer N., in't Zand J. J. M., 2009, A\&A, 502, 871

Linares M., Altamirano D., Chakrabarty D., Cumming A., Keek L., 2012, ApJ, 748, 82

Linares M., Altamirano D., Watts A., van der Klis M., Wijnands R., Homan J., Casella P., Patruno A., Armas-Padilla M., Cavecchi Y., Degenaar N., Kalamkar M., Kaur R., Yang Y., Rea N., 2010, The Astronomer's Telegram, 2958, 1

Liu Q. Z., van Paradijs J., van den Heuvel E. P. J., 2007, A\&A, 469, 807

Lomb N. R., 1976, Ap\&SS, 39, 447

Lyu M., Méndez M., Zhang G., Keek L., 2015, MNRAS, 454, 541

Mahmoodifar S., Strohmayer T., 2015, ArXiv e-prints

Maurer I., Watts A. L., 2008, MNRAS, 383, 387

Muno M. P., Fox D. W., Morgan E. H., Bildsten L., 2000, ApJ, 542, 1016

Press W. H., Teukolsky S. A., Vetterling W. T., Flannery B. P., 2002, Numerical recipes in $\mathrm{C}++$ : the art of scientific computing 


\section{Ming Lyu et al.}

Revnivtsev M., Churazov E., Gilfanov M., Sunyaev R., 2001, A\&A, 372, 138

Scargle J. D., 1982, ApJ, 263, 835

Strohmayer T. E., Markwardt C. B., 2002, ApJ, 577, 337

van der Klis M., 2006, in Compact Stellar X-Ray Sources, ed. W. H. G. Lewin \& M. van der Klis (Cambridge: Cambridge Univ. Press)

van Paradijs J., Penninx W., Lewin W. H. G., 1988, MNRAS, 233, 437

van Straaten S., van der Klis M., di Salvo T., Belloni T., 2002, ApJ, 568, 912

van Straaten S., van der Klis M., Wijnands R., 2005, ApJ, 619, 455

Wijnands R., 2001, ApJ, 554, L59

Zhang G., Méndez M., Altamirano D., 2011, MNRAS, 413, 1913

Zhang G., Méndez M., Zamfir M., Cumming A., 2016, MNRAS, 455, 2004 